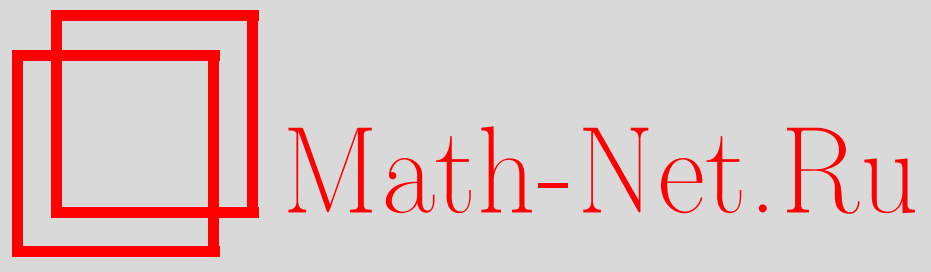

В. А. Франке, Разные канонические формулировки теории гравитации Эйнштейна, ТMФ, 2006, том 148, номер 1, 143-160

DOI: https://doi.org/10.4213/tmf2065

Использование Общероссийского математического портала Math-Net.Ru подразумевает, что вы прочитали и согласны с пользовательским соглашением http://www . mathnet.ru/rus/agreement

Параметры загрузки:

IP : 34.227 .88 .159

26 апреля 2023 г., 18:03:03

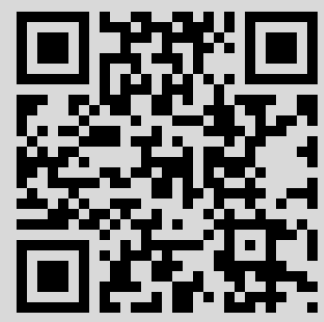




\title{
РАЗНЫЕ КАНОНИЧЕСКИЕ ФОРМУЛИРОВКИ ТЕОРИИ ГРАВИТАЦИИ ЭЙНШТЕЙНА
}

\begin{abstract}
Описаны четыре наиболее известных варианта классического канонического формализма в теории гравитации Эйнштейна: формализмы АрновиттаДезера-Мизнера, Фаддеева-Попова, реперный формализм в обычном виде и в виде, приспособленном к построению петлевой теории гравитации, которая разрабатывается в настоящее время. Приведены канонические преобразования, связывающие эти формализмы.
\end{abstract}

Ключевые слова: теория гравитации, канонический формализм.

\section{1. ВВЕДЕНИЕ}

Наиболее прямой путь к построению квантовой теории - квантование соответствующей классической теории, записанной в канонической форме. Разные эквивалентные канонические формулировки классической теории могут при этом приводить к не вполне эквивалентным вариантам квантовой теории. Поэтому в сложных случаях полезно до квантования представить классическую теорию в канонической форме многими способами. Это, в частности, относится к теории гравитации, окончательная квантовая форма которой пока не найдена. Не исключено, что, подобрав подходящую классическую каноническую формулировку, здесь можно приблизиться к удовлетворительному решению задачи квантования. Именно такой подход лежит в основе так называемой петлевой теории гравитации (см. обзор [1] и библиографию в нем), которая разрабатывается в настоящее время.

Данная статья, преследующая в основном педагогические цели, посвящена описанию нескольких известных эквивалентных классических канонических формулировок теории гравитации Эйнштейна и связей между этими формулировками. Сначала излагается формализм Арновитта-Дезера-Мизнера (АДМ) [2]. Затем с помощью канонического преобразования осуществляется переход к формализму ФаддееваПопова (ФП) [3]. Далее посредством замены переменных вводится реперный формализм в обычном виде. Наконец, путем канонического преобразования этот формализм приводится к виду, лежащему в основе петлевой теории гравитации [1].

${ }^{*}$ Санкт-Петербургский государственный университет, Санкт-Петербург, Россия. E-mail: franke@pobox.spbu.ru 
Мы не рассматриваем здесь проблемы квантования гравитации, ограничиваясь лишь отдельными замечаниями по этому поводу, но изложенные сведения могут быть полезны при работе над данной проблемой.

\section{2. ФОРМАЛИЗМ АДМ}

Остановимся, прежде всего, на классическом формализме АДМ [2]. Пусть $x^{\mu-}$ координаты в римановом пространстве-времени $(\mu, \nu, \ldots=0,1,2,3)$. Координату $x^{0}=t$ именуем временем (принимая $c=1$, где $c$ - скорость света). Считаем, что все гиперповерхности $x^{0}=$ const пространственноподобны. Пространственные координаты обозначаем через $x^{i}(i, k, \ldots=1,2,3)$. Используем сигнатуру метрики $(-,+,+,+)$.

Фиксируем некоторую гиперповерхность $x^{0}=$ const и обозначим ее через $\Sigma$. В координатах $x^{i}$ трехмерная метрика, индуцируемая на $\Sigma$, совпадает с трехмерной частью четырехмерной метрики. Обозначим эту трехмерную метрику через $\beta_{i k}$ и определим $\beta^{i k}$ условием

$$
\beta_{i k} \beta^{k l}=\delta_{i}^{l}
$$

Тогда

$$
\begin{aligned}
& \beta_{i k}=g_{i k}, \\
& \beta^{i k}=g^{i k}-\frac{g^{0 i} g^{0 k}}{g^{00}},
\end{aligned}
$$

где $g_{\mu \nu}$ - четырехмерная метрика, $g^{\mu \nu} g_{\mu \lambda}=\delta_{\lambda}^{\mu}$.

Введем обозначения

$$
g=\operatorname{det} g_{\mu \nu}, \quad \beta=\operatorname{det} \beta_{i k} .
$$

Как обычно,

$$
\begin{gathered}
R_{\beta, \gamma \delta}^{\alpha}=\partial_{\gamma} \Gamma_{\delta \beta}^{\alpha}-\partial_{\delta} \Gamma_{\gamma \beta}^{\alpha}+\Gamma_{\gamma \rho}^{\alpha} \Gamma_{\delta \beta}^{\rho}-\Gamma_{\delta \rho}^{\alpha} \Gamma_{\gamma \beta}^{\rho}, \\
R_{\beta \delta}=R^{\alpha}{ }_{\beta, \alpha \delta}, \\
R=g^{\beta \delta} R_{\beta \delta}
\end{gathered}
$$

где $\Gamma_{\beta \gamma}^{\alpha}-$ символы Кристоффеля, построенные из метрики $g_{\mu \nu}$ известным образом. Определим величины $\stackrel{(3)}{\Gamma}_{k l}^{i}, \stackrel{(3)}{R}_{k, l m}^{i}, \stackrel{(3)}{R}_{l m}, \stackrel{(3)}{R}$, образованные из $\beta_{i k}, \partial_{l} \beta_{i k}, \partial_{m} \partial_{l} \beta_{i k}$ точно так же, как $\Gamma_{\beta \gamma}^{\alpha}, R_{\beta, \gamma \delta}^{\alpha}, R_{\beta \delta}, R$ построены из $g_{\mu \nu}, \partial_{\alpha} g_{\mu \nu}, \partial_{\alpha} \partial_{\beta} g_{\mu \nu}$. Введем ковариантную производную $\stackrel{(3)}{\nabla}$, действующую на $\Sigma$ при помощи связности $\stackrel{(3)}{\Gamma} \underset{k l}{i}$ так же, как производная $\nabla_{\mu}$ действует на всем пространстве-времени с помощью связности $\Gamma_{\beta \gamma}^{\alpha}$. Определим второй фундаментальный тензор $K_{i k}$ гиперповерхности $\Sigma$ :

$$
K_{i k}=K_{k i}=-\left.\nabla_{i} n_{k}\right|_{\Sigma}=\left.n_{0} \Gamma_{i k}^{0}\right|_{\Sigma}=-\left.\frac{1}{\sqrt{-g^{00}}} \Gamma_{i k}^{0}\right|_{\Sigma},
$$


где поле $n_{\mu}(x)$ единичных нормалей к поверхностям $x^{0}=$ const задается равенствами

$$
n_{\mu} n^{\mu}=-1, \quad n_{\mu}=-\delta_{\mu}^{0} \frac{1}{\sqrt{-g^{00}}}, \quad n^{\mu}=-\frac{g^{0 \mu}}{\sqrt{-g^{00}}} .
$$

Имеет место тождество

$$
\sqrt{-g} R=\sqrt{-g}\left(\stackrel{(3)}{R}+K_{l}^{i} K_{i}^{l}-\left(K_{i}^{i}\right)^{2}\right)+2 \partial_{\gamma}\left(\sqrt{-g}\left(n^{\gamma} \nabla_{\delta} n^{\delta}-n^{\delta} \nabla_{\delta} n^{\gamma}\right)\right) .
$$

Здесь $K_{l}^{i}=\beta^{i k} K_{k l}$. Достаточно простой вывод этого тождества основан на известной формуле Гаусса, связывающей тензор кривизны гиперповерхности с тензором кривизны объемлющего риманова пространства.

Рассматриваем только гравитационное поле, не взаимодействующее с другими полями, поскольку уже в этом случае видны все особенности задачи. Исходим из действия гравитационного поля

$$
S=\int d^{4} x \mathcal{L}
$$

где

$$
\mathcal{L}=\frac{1}{2 \varkappa} \sqrt{-g}\left(g^{\alpha \beta}\left(\Gamma_{\alpha \gamma}^{\rho} \Gamma_{\rho \beta}^{\gamma}-\Gamma_{\alpha \beta}^{\rho} \Gamma_{\rho \gamma}^{\gamma}\right)-2 \Lambda\right) .
$$

Здесь $\varkappa=8 \pi \gamma, \gamma$ - ньютонова гравитационная постоянная, $\Lambda$ - космологическая постоянная. Иначе,

$$
\mathcal{L}=\frac{1}{2 \varkappa} \sqrt{-g}(R-2 \Lambda)+\frac{1}{2 \varkappa} \partial_{\gamma}\left(\sqrt{-g}\left(g^{\gamma \alpha} \Gamma_{\alpha \beta}^{\beta}-g^{\alpha \beta} \Gamma_{\alpha \beta}^{\gamma}\right)\right)
$$

С помощью тождества (10) находим отсюда, что

$$
\begin{aligned}
\mathcal{L}= & \frac{1}{2 \varkappa} \sqrt{-g}\left(\stackrel{(3)}{R}+K_{l}^{i} K_{i}^{l}-\left(K_{i}^{i}\right)^{2}-2 \Lambda\right)+\frac{1}{2 \varkappa} \partial_{\gamma}\left(\sqrt{-g}\left(g^{\gamma \alpha} \Gamma_{\alpha \beta}^{\beta}-g^{\alpha \beta} \Gamma_{\alpha \beta}^{\gamma}\right)\right)+ \\
& +2 \partial_{\gamma}\left(\sqrt{-g}\left(n^{\gamma} \nabla_{\delta} n^{\delta}-n^{\delta} \nabla_{\delta} n^{\gamma}\right)\right) .
\end{aligned}
$$

В случае замкнутой Вселенной здесь можно отбросить дивергенцию, а в случае островного расположения масс в асимптотически трехмерно-плоском пространствевремени достаточно учесть лишь существенную часть дивергенции, равную

$$
\frac{1}{2 \varkappa}\left(\partial_{k} \partial_{k} \beta_{l l}-\partial_{i} \partial_{k} \beta_{i k}\right)
$$

причем в последнем случае $\Lambda=0$.

Далее мы часто для простоты отбрасываем дивергенцию, считая Вселенную замкнутой, а также нередко опускаем $\Lambda$-член.

В качестве независимых полевых переменных АДМ выбирают величины

$$
\beta_{i k} \equiv g_{i k}, \quad N \equiv \frac{1}{\sqrt{-g^{00}}}, \quad N_{i}=g_{0 i} .
$$


В дальнейшем индексы $i, k, \ldots$ поднимаются и опускаются с помощью трехмерных тензоров $\beta^{i k}$ и $\beta_{i k}$.

Справедливы равенства

$$
\begin{gathered}
g_{i k}=\beta_{i k}, \quad g^{i k}=\beta^{i k}-\frac{N^{i} N^{k}}{N^{2}}, \quad g_{0 k}=N_{k}, \quad g^{0 k}=\frac{N^{k}}{N^{2}} \\
g_{00}=-N^{2}+N_{k} N^{k}, \quad g^{00}=-\frac{1}{N^{2}}, \quad \sqrt{-g}=N \sqrt{\beta} \\
n_{\mu}=-\delta_{\mu}^{0} N, \quad n^{0}=\frac{1}{N}, \quad n^{i}=-\frac{N^{i}}{N} \\
K_{i k}=\frac{1}{2 N}\left(\stackrel{(3)}{\nabla}_{i} N_{k}+\stackrel{(3)}{\nabla}_{k} N_{i}-\partial_{0} \beta_{i k}\right) .
\end{gathered}
$$

В этих переменных плотность лагранжиана (14) с отброшенной дивергенцией принимает вид

$$
\mathcal{L}^{(\text {АДМ })}=N\left\{2 f^{i j, k l} K_{i j} K_{k l}+\frac{\sqrt{\beta}}{2 \varkappa}(\stackrel{(3)}{R}-2 \Lambda)\right\}
$$

где

$$
g^{i j, k l}=\frac{1}{4}\left(\frac{\sqrt{\beta}}{2 \varkappa}\right)\left(\beta^{i k} \beta^{j l}+\beta^{i l} \beta^{j k}-2 \beta^{i j} \beta^{k l}\right) .
$$

Удобно ввести символы

$$
\delta_{l m}^{i k} \equiv \frac{1}{2}\left(\delta_{l}^{i} \delta_{m}^{k}+\delta_{m}^{i} \delta_{l}^{k}\right)
$$

и определить величину $\mathcal{J}_{i j, k l}$ с помощью условия

$$
\jmath^{i j, k l} \jmath_{k l, m n}=\delta_{n m}^{i j} .
$$

При этом

$$
\partial_{i j, k l}=\left(\frac{2 \varkappa}{\sqrt{\beta}}\right)\left(\beta_{i k} \beta_{j l}+\beta_{i l} \beta_{j k}-\beta_{i j} \beta_{k l}\right), \quad \mathcal{J}_{i j, k l}=\mathcal{J}_{k l, i j}=\mathcal{J}_{j i, k l}=\mathcal{J}_{i j, l k}
$$

и аналогично для $\mathrm{g}^{i j, k l}$.

В выражении для $\mathcal{L}$ мы вновь отбросили возникающую несущественную добавку к дивергенции.

Полагая

$$
L=\int_{x^{0}=\text { const }} d^{3} x \mathcal{L}^{(\text {АДМ })},
$$

определяем сопряженные импульсы:

$$
\begin{aligned}
P^{(N)}(x) & \equiv \frac{\delta L}{\delta\left(\partial_{0} N(x)\right)}, \\
P^{\left(N_{i}\right)}(x) & \equiv \frac{\delta L}{\delta\left(\partial_{0} N_{i}(x)\right)}, \\
P^{i k}(x) & \equiv \frac{\delta L}{\delta\left(\partial_{0} \beta_{i k}(x)\right)}=\frac{\partial \mathcal{L}(\text { АДМ })}{\partial\left(\partial_{0} \beta_{i k}(x)\right)},
\end{aligned}
$$


где $x \equiv\left(x^{1}, x^{2}, x^{3}\right)$, а $\delta / \delta(\ldots)$ - трехмерная вариационная производная. Сразу же находим первичные связи:

$$
P^{(N)}(x)=0, \quad P^{\left(N_{i}\right)}(x)=0 .
$$

Эти связи учитываем явно, т.е. полагаем $P^{(N)}$ и $P^{\left(N_{i}\right)}$ равными нулю везде, где они встречаются.

Далее,

$$
P^{i k}(x)=\frac{\partial \mathcal{L}^{(\mathrm{AДМ)})}}{\partial K_{l m}} \frac{\partial K_{l m}}{\partial\left(\partial_{0} \beta_{i k}\right)}=-2 \partial^{i k, l m} K_{l m},
$$

откуда

$$
K_{i k}=-\frac{1}{2} \mathcal{\partial}_{i k, l m} P^{l m},
$$

причем согласно (20)

$$
\partial_{0} \beta_{i k}=\stackrel{(3)}{\nabla}_{i} N_{k}+\stackrel{(3)}{\nabla}_{k} N_{i}-2 N K_{i k} .
$$

Плотность обобщенного гамильтониана равна

$$
\mathcal{H}^{(\text {gen })}=P^{i k} \partial_{0} \beta_{i k}-\mathcal{L}^{(\mathrm{AДM})} .
$$

Вновь отбрасывая несущественную добавку к дивергенции, находим, что

$$
\mathcal{H}^{\text {(gen) }}=N \mathcal{H}_{0}+N^{i} \mathcal{H}_{i},
$$

где

$$
\begin{gathered}
\mathcal{H}_{0}=\frac{1}{2} \mathcal{J}_{i k, l m} P^{i k} P^{l m}+\left(\frac{\sqrt{\beta}}{2 \varkappa}\right)(-\stackrel{(3)}{R}+2 \Lambda), \\
\mathcal{H}_{i}=-2 \beta_{i s} \sqrt{\beta} \stackrel{(3)}{\nabla}_{l}\left(\frac{P^{l s}}{\sqrt{\beta}}\right) .
\end{gathered}
$$

Учтено, что $P^{l s} / \sqrt{\beta}$ есть тензор.

Плотность лагранжиана первого порядка равна

$$
\mathcal{L}_{(1)}^{(\mathrm{AДМ)}}=P^{i k} \partial_{0} \beta_{i k}-\mathcal{H}^{(\text {gen })}=P^{i k} \partial_{0} \beta_{i k}-N \mathcal{H}_{0}-N^{i} \mathcal{H}_{i} .
$$

Добавляя существенную часть дивергенции, имеем для островного расположения масс в асимптотически трехмерно-плоском пространстве

$$
\mathcal{L}_{(1)}^{(\mathrm{AДM})}=P^{i k} \partial_{0} \beta_{i k}-\mathcal{H}^{(\mathrm{gen})}=P^{i k} \partial_{0} \beta_{i k}-N \mathcal{H}_{0}-N^{i} \mathcal{H}_{i}-\frac{1}{2 \varkappa}\left(\partial_{i} \partial_{k} \beta_{i k}-\partial_{k} \partial_{k} \beta_{i i}\right) .
$$

Варьируя $\mathcal{L}_{(1)}^{(\text {Адм) }}$ по $N$ и $N^{i}$, получаем вторичные связи:

$$
\mathcal{H}_{0}(x)=0, \quad \mathcal{H}_{i}(x)=0 .
$$

В случае островного расположения масс полная энергия сводится к поверхностному интегралу

$$
H^{(\text {полн })}=\frac{1}{2 \varkappa} \int d^{3} x\left(\partial_{i} \partial_{k} \beta_{i k}-\partial_{k} \partial_{k} \beta_{i i}\right),
$$


причем теперь

$$
\mathcal{H}^{\text {(gen })}=N \mathcal{H}_{0}+N^{i} \mathcal{H}_{i}+\frac{1}{2 \varkappa}\left(\partial_{i} \partial_{k} \beta_{i k}-\partial_{k} \partial_{k} \beta_{i i}\right) .
$$

В случае замкнутой Вселенной полная энергия равна нулю.

Вводим скобки Пуассона. Если $F_{1}$ и $F_{2}$ - два трехмерных функционала от $\beta_{i k}$, $P^{i k}$, то

$$
\left\{F_{1}, F_{2}\right\}=\int d^{3} x\left(\frac{\delta F_{1}}{\delta \beta_{i k}(x)} \frac{\delta F_{2}}{\delta P^{i k}(x)}-\frac{\delta F_{2}}{\delta \beta_{i k}(x)} \frac{\delta F_{1}}{\delta P^{i k}(x)}\right),
$$

где $\delta / \delta(\ldots)$ - трехмерная вариационная производная. Очевидно,

$$
\begin{gathered}
\left\{F_{1}, F_{2}\right\}=-\left\{F_{2}, F_{1}\right\}, \\
\left\{F_{1},\left\{F_{2}, F_{3}\right\}\right\}+\left\{F_{2},\left\{F_{3}, F_{1}\right\}\right\}+\left\{F_{3},\left\{F_{1}, F_{2}\right\}\right\}=0, \\
\left\{F_{1}, F_{2} F_{3}\right\}=\left\{F_{1}, F_{2}\right\} F_{3}+F_{2}\left\{F_{1}, F_{3}\right\} .
\end{gathered}
$$

Далее применяем обозначения

$$
f \equiv f(x), \quad \underset{\sim}{f} \equiv f(\tilde{x}) .
$$

В этих обозначениях

$$
\begin{gathered}
\left\{\beta_{i k}, \underset{\sim}{\left.P^{l m}\right\}}=\delta_{i k}^{l m} \delta^{3}(x-\tilde{x}),\right. \\
\left\{\beta_{i k}, \underset{\sim}{\beta_{l m}}\right\}=0, \quad\left\{P^{i k}, \underset{\sim}{P^{l m}}\right\}=0 .
\end{gathered}
$$

Справедливы соотношения

$$
\begin{aligned}
\left\{\mathcal{H}_{i}, \underset{\sim}{\mathcal{H}_{k}}\right\} & =\mathcal{H}_{k} \partial_{i} \delta^{3}(x-\tilde{x})-\underset{\sim}{\mathcal{H}_{i} \partial_{j}} \delta^{3}(x-\tilde{x}), \\
\left\{\mathcal{H}_{i}, \underset{\sim}{\mathcal{H}_{0}}\right\} & =\mathcal{H}_{0} \partial_{i} \delta^{3}(x-\tilde{x}), \\
\left\{\mathcal{H}_{0}, \underset{\sim}{\mathcal{H}_{0}}\right\} & =\beta^{i k} \mathcal{H}_{k} \partial_{i} \delta^{3}(x-\tilde{x})-\underset{\sim}{\beta^{i k}} \underset{\sim}{\mathcal{H}_{k}} \partial_{\sim} \delta^{3}(x-\tilde{x}),
\end{aligned}
$$

где $\underset{\sim}{\partial_{i}}=\partial / \partial \tilde{x}^{i}$. Видно, что в классике все связи первого рода. Никаких новых связей не возникает.

Связи $\mathcal{H}_{i}$ являются генераторами трехмерных преобразований координат на поверхности $\Sigma$. Действительно, при замене координат

$$
x^{i} \rightarrow x^{i}+\xi^{i}(x),
$$

где $\xi^{i}(x)$ бесконечно малы, имеем

$$
\begin{aligned}
\delta \beta_{i k} & \equiv \beta_{i k}^{\prime}(x)-\beta_{i k}(x)=-\stackrel{(3)}{\nabla}_{i} \xi_{k}-\stackrel{(3)}{\nabla}_{k} \xi_{i}, \\
\delta P^{i k} & \equiv P^{\prime i k}(x)-P^{i k}(x)=\left(\partial_{l} \xi^{i}\right) P^{l k}+P^{i l} \partial_{l} \xi^{k}-\partial_{l}\left(P^{i k} \xi^{l}\right) .
\end{aligned}
$$

Можно непосредственно проверить, что

$$
\left\{\int d^{3} x \mathcal{H}_{i} \xi^{i}, \underset{\sim}{\beta_{k l}}\right\}=\underset{\sim}{\delta \beta_{k l},} \quad\left\{\int d^{3} x \mathcal{H}_{i} \xi^{i}, \underset{\sim}{P^{k l}}\right\}=\underset{\sim}{\underset{\sim}{P^{k l}} .}
$$


Соответственно связь $\mathcal{H}_{0}$ генерирует сдвиги точек поверхности $\Sigma$ вдоль нормалей к $\Sigma$. Изменения величин $\beta_{i k}$ и $P^{i k}$ при этом соответствуют решениям уравнений Эйнштейна.

Сделаем лишь отдельные замечания относительно квантования описанной теории.

При квантовании переменные $\beta_{i k}, P^{i k}$ заменяются операторами, подчиненными условиям

$$
\begin{aligned}
& {\left[\beta_{i k}, \underset{\sim}{P^{l m}}\right]=i \delta_{i k}^{l m} \delta^{3}(x-\tilde{x}),} \\
& {\left[\beta_{i k}, \underset{\sim}{\beta_{l m}}\right]=\left[P^{i k}, \underset{\sim}{P^{l m}}\right]=0 .}
\end{aligned}
$$

Поскольку связи (36), (37) слишком сложны, чтобы их можно было решить явно, эти связи обычно налагают на вектор состояния. Возникающая теория оказывается корректной только при условии, что коммутаторы связей друг с другом равны линейным комбинациям этих связей с коэффициентами, стоящими слева от них. После квантования связи удовлетворяют коммутационным соотношениям вида (50)-(52) с заменой скобок \{\} на $-i[$ ]. Однако теперь играет роль выбранный порядок множителей $\beta_{i k}$ и $P^{i k}$ в выражениях для связей. Может случиться, что результат коммутирования связей содержит эти множители не в том порядке, который был принят в связях первоначально, а коэффициенты при связях могут возникать не только слева от них. Легко убедиться, что в квантовых аналогах соотношений (50), (51) этого не происходит, и указанные соотношения после квантования сохраняют свой вид (с точностью до замены \{\}$\rightarrow-i[])$. Последнее, в частности, обусловлено отмеченным геометрическим смыслом связей $\mathcal{H}_{i}$ как генераторов преобразований трехмерных координат. Этот смысл полностью сохраняется после квантования.

Иначе обстоит дело с квантовым аналогом соотношения (52). Если операторы в связях $\mathcal{H}_{0}, \mathcal{H}_{i}$ расположены так, что эти связи эрмитовы, то величина $-i\left[\mathcal{H}_{0}, \underset{\sim}{\mathcal{H}_{0}}\right]$, в которую превращается $\left\{\mathcal{H}_{0}, \mathcal{H}_{0}\right\}$, тоже будет эрмитовой. Значит, справа в аналоге равенства (52) не могут возникнуть неэрмитовы выражения $\beta^{i k} \mathcal{H}_{k}$ (мы учитываем, что $\beta^{i k}$ и $\mathcal{H}_{k}$ не коммутируют). Самое большее, что можно получить для коммутатора $-i\left[\mathcal{H}_{0}, \mathcal{H}_{0}\right]$, подбирая порядок множителей в $\mathcal{H}_{0}$ и $\mathcal{H}_{k}$ без нарушения эрмитовости, - это выражжение вида

$$
\begin{gathered}
\frac{1}{2}\left(\beta^{i k} \mathcal{H}_{k}+\mathcal{H}_{k} \beta^{k i}\right) \partial_{i} \delta^{3}(x-\tilde{x})-\frac{1}{2}\left(\underset{\sim}{\beta_{\sim}^{i k}} \underset{\sim}{\mathcal{H}_{k}}+\underset{\sim}{\mathcal{H}_{k}} \beta_{\sim}^{k i}\right) \underset{\sim}{\partial_{i} \delta^{3}}(x-\tilde{x})= \\
=\beta^{i k} \mathcal{H}_{k} \partial_{i} \delta^{3}(x-\tilde{x})-\underset{\sim}{\beta^{i k}} \underset{\sim}{\mathcal{H}_{k}} \partial_{i} \delta^{3}(x-\tilde{x})+ \\
\quad+\delta^{3}(0)(\ldots) \partial_{i} \delta^{3}(x-\tilde{x})-\delta^{3}(0)(\ldots) \underset{\sim}{\partial_{i}} \delta^{3}(x-\tilde{x}),
\end{gathered}
$$

где $(\ldots),(\ldots)$ - некоторые операторнозначные функции, не равные нулю. Символы $\delta^{3}(0)$ возникают из-за коммутирования операторов $\beta^{i k}, \mathcal{H}_{k}$ или $\beta_{\sim}^{i k}, \mathcal{H}_{\sim}$, взятых в одной точке. 
Ясно, что выражение вида (59), содержащее произведение $\delta^{3}(0) \partial_{i} \delta^{3}(x-\tilde{x})$, бессмысленно. Придать ему смысл можно только путем регуляризации. Вопрос заключается в том, можно ли так выбрать регуляризацию, чтобы лишние члены в выражении (59) обратились в нуль, а после снятия регуляризации восстановилась общековариантность теории. Однозначный ответ на этот вопрос до сих пор не получен. В ряде имеющихся работ проблема регуляризации и ее снятия изучена недостаточно строго. Такое положение обусловлено тем, что методы регуляризации подробно разработаны только в рамках теории возмущений. Здесь же задача поставлена вне этих рамок.

Несмотря на неясность, сохраняющуюся в данном вопросе, было проведено квантование теории гравитации методом континуального интеграла по аналогии с квантованием неабелевых калибровочных теорий (см. работу [3] и библиографию в ней, а также книгу [4]). Если бы таким путем была получена удовлетворительная теория возмущений, то ее корректность можно было бы проверить непосредственно в рамках диаграммного формализма Фейнмана, и этого было бы достаточно. Но оказалось, что построенная теория возмущений неперенормируема. В связи с этим сейчас разрабатываются другие подходы к построению квантовой теории гравитации, наиболее известные из которых - теория суперструн (см., например, книгу [5]) и так называемая петлевая теория гравитации [1].

Заметим также, что отмеченные трудности с замыканием алгебры связей после квантования характерны и для других вариантов канонического формализма в теории гравитации, описанных ниже.

\section{3. ФОРМАЛИЗМ ФП}

Остановимся теперь на классическом каноническом формализме ФП [3]. Прежде всего, вводятся величины

$$
h^{\mu \nu}=\sqrt{-g} g^{\mu \nu},
$$

в терминах которых дополнительное гармоническое координатное условие записывается особенно просто: $\partial_{\mu} h^{\mu \nu}=0$. В качестве исходных переменных выбираются функции

$$
q^{i k} \equiv h^{0 i} h^{k 0}-h^{00} h^{i k},
$$

причем далее $q \equiv \operatorname{det} q^{i k}$. Кроме того, сохраняются функции $N$ и $N^{i}$, имевшиеся в формализме АДМ.

Следующее каноническое преобразование связывает формализмы АДМ и ФП:

$$
\begin{aligned}
& \left\{\begin{array}{l}
q^{i k}=\beta \beta^{i k}=\frac{1}{2} \varepsilon^{i l m} \varepsilon^{k n p} \beta_{l n} \beta_{m p}, \\
\pi_{i k}=-\frac{1}{(2 \varkappa) 2 \sqrt{\beta}} \mathcal{J}_{i k, l m} P^{l m}=\beta^{-1}\left(\frac{1}{2} \beta_{i k} \beta_{l m}-\beta_{i l} \beta_{k m}\right) P^{l m},
\end{array}\right. \\
& \left\{\begin{array}{l}
\beta_{i k}=\frac{1}{2 \sqrt{q}} \varepsilon_{i l m} \varepsilon_{k n p} q^{l n} q^{m p}, \\
P^{l m}=-\frac{1}{\sqrt{q}}\left(q^{i k} q^{l m}-q^{l i} q^{m k}\right) \pi_{i k},
\end{array}\right.
\end{aligned}
$$


где $\pi_{i k}$ - импульсы, сопряженные обобщенным координатам $q^{i k}, \varepsilon_{i k l}$ - вполне антисимметричный символ, $\varepsilon_{123}=1$. При этом

$$
\begin{gathered}
\pi_{i k} \partial_{0} q^{i k}=P^{i k} \partial_{0} \beta_{i k}, \\
\left\{q^{i k},{\underset{\sim}{l m}}_{l m}\right\}=\delta_{l m}^{i k} \delta^{3}(x-\tilde{x}) \\
\left\{q^{i k},{\left.\underset{\sim}{q^{l m}}\right\}=0, \quad\left\{\pi_{i k},{\underset{\sim}{l m}}_{l m}\right\}=0 .}^{\pi^{l m}}=0,\right.
\end{gathered}
$$

В формализме ФП плотность лагранжиана первого порядка имеет вид

$$
\mathcal{L}_{1}^{(\Phi \Pi)}=\pi_{i k} \partial_{0} q^{i k}-N \mathcal{H}_{0}-N^{i} \mathcal{H}_{i}+\frac{1}{2 \varkappa} \partial_{i} \partial_{k} q^{i k},
$$

где выписана часть дивергенции, существенная в случае островного расположения масс в асимптотически трехмерно-плоском пространстве-времени. Теперь

$$
\begin{aligned}
\mathcal{H}_{0} & =\frac{2 \varkappa}{q^{1 / 4}}\left(q^{l p} q^{m q}-q^{l m} q^{p q}\right) \pi_{l m} \pi_{p q}-\frac{q^{1 / 4}}{2 \varkappa}(\stackrel{(3)}{R}-2 \Lambda), \\
\mathcal{H}_{i} & =\frac{2}{q^{1 / 4}} q^{k l}\left(\stackrel{(3)}{\nabla}{ }_{k}\left(q^{1 / 4} \pi_{i l}\right)-\stackrel{(3)}{\nabla}_{i}\left(q^{1 / 4} \pi_{k l}\right)\right),
\end{aligned}
$$

(3)

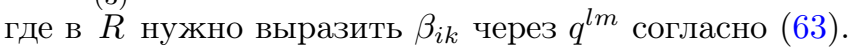

Величины $\mathcal{H}_{0}, \mathcal{H}_{i}$ по-прежнему удовлетворяют соотношениям (50)-(52), причем геометрический смысл этих величин сохраняется.

\section{4. ОБЫЧНЫЙ РЕПЕРНЫЙ ФОРМАЛИЗМ}

Обратимся теперь к реперному формализму. Введем в каждой точке пространства-времени четыре взаимно псевдоортогональных нормированных вектора $e_{A}^{\mu}(x)$, где индекс $A$ нумерует векторы $(A, B, \ldots=0,1,2,3)$, а индекс $\mu$ - их компоненты в координатном базисе $(\mu, \nu, \ldots=0,1,2,3)$, причем $x \equiv\left\{x^{0}, x^{1}, x^{2}, x^{3}\right\}$. Предполагается, что

$$
e_{A}^{\mu}(x) g_{\mu \nu} e_{B}^{\nu}(x)=\eta_{A B},
$$

где $\eta_{A B}=\operatorname{diag}(-1,1,1,1)$. Определим также величины $e_{\mu}^{A}(x)$ равенством

$$
e_{\mu}^{A} e_{A}^{\nu}=\delta_{\mu}^{\nu}
$$

Из соотношений (70), (71) следует, что

$$
g_{\mu \nu}(x)=e_{\mu}^{A} \eta_{A B} e_{\nu}^{B} .
$$

Подставим это выражение в приведенное ранее действие гравитационного поля (11), $(12)$ и будем рассматривать далее $e_{\mu}^{A}(x)$ как функции, описывающие это поле. Возникнет теория, инвариантная относительно двух групп преобразований: общих преобразований координат и лоренцевых локальных преобразований величин $e_{\mu}^{A}(x)$. Последние преобразования имеют вид

$$
e_{\mu}^{A}(x)=\omega_{B}^{A}(x) e_{\mu}^{B}(x),
$$


причем

$$
\eta_{A B} \omega^{A}{ }_{D}(x) \omega^{B}{ }_{E}(x)=\eta_{D E} .
$$

Равенство (74) обеспечивает неизменность метрики $g_{\mu \nu}(x)$ при таких преобразованиях. Величины $e_{\mu}^{A}, e_{A}^{\mu}$ именуем далее реперными параметрами.

Каждому вектору, отнесенному к координатному базису, сопоставляется вектор, отнесенный к реперному, по правилу

$$
a^{A}=e_{\mu}^{A} a^{\mu}, \quad a_{A}=e_{A}^{\mu} a_{\mu} .
$$

Аналогично можно определить тензоры $T_{\nu, \ldots, B, \ldots}^{\mu, \ldots, A, \ldots}$, которые при замене координат изменяются обычным образом, по индексам $\mu, \ldots, \nu, \ldots$, а при замене параметров $e^{A} \mu$ согласно равенству (73) преобразуются лоренцевыми матрицами по индексам $A, \ldots$, $B, \ldots$ Индексы $A, \ldots, B, \ldots$ у тензоров поднимаются и опускаются с помощью величин $\eta^{A B}$ и $\eta_{A B}$.

Как обычно, определяются ковариантные производные

$$
\begin{aligned}
\nabla_{\mu} a^{\nu} & =\partial_{\mu} a^{\nu}+\Gamma_{\mu \alpha}^{\nu} a^{\alpha}, & \nabla_{\mu} a_{\nu} & =\partial_{\mu} a_{\nu}-a_{\alpha} \Gamma_{\mu \nu}^{\alpha}, \\
\nabla_{\mu} a^{A} & =\partial_{\mu} a^{A}+A_{\mu}{ }^{A}{ }_{B} a^{B}, & \nabla_{\mu} a_{A} & =\partial_{\mu} a_{A}-a_{B} A_{\mu}{ }^{B}{ }_{A}
\end{aligned}
$$

(и аналогично для тензоров). Требуя выполнения равенств

$$
\nabla_{\mu} e_{\nu}^{A}=0, \quad \nabla_{\mu} e_{A}^{\nu}=0, \quad \nabla_{\mu} \eta^{A B}=0,
$$

устанавливаем связь между $\Gamma_{\mu \nu}^{\alpha}$ и $A_{\mu}{ }^{A}{ }_{B}$ :

$$
\begin{gathered}
\Gamma_{\alpha \nu}^{\mu}=e_{A}^{\mu} A_{\alpha}{ }^{A}{ }_{B} e_{\nu}^{B}+e_{A}^{\mu} \partial_{\alpha} e_{\nu}^{A}, \\
A_{\alpha}{ }^{A}{ }_{B}=e_{\mu}^{A} \Gamma_{\alpha \nu}^{\mu} e_{B}^{\nu}+e_{\mu}^{A} \partial_{\alpha} e_{B}^{\mu} .
\end{gathered}
$$

Именуем $A_{\alpha}{ }^{A}$ B реперной связностью, а $\Gamma_{\alpha \nu}^{\mu}$ - координатной связностью.

Реперная связность аналогична калибровочному полю со структурной группой Лоренца. Поэтому

$$
A_{\alpha}{ }^{A}{ }_{B}=A_{\alpha}{ }^{A D} \eta_{D B}
$$

где $A_{\alpha}{ }^{A D}=-A_{\alpha}{ }^{D A}$. Понимая под $A_{\alpha}$ матрицу $A_{\alpha}{ }_{B}$, можем составить аналог напряженности поля

$$
F_{\mu \nu}=\partial_{\mu} A_{\nu}-\partial_{\nu} A_{\mu}+A_{\mu} A_{\nu}-A_{\nu} A_{\mu}
$$

причем

$$
R_{\beta, \mu \nu}^{\alpha}=e_{A}^{\alpha} F_{\mu \nu}{ }^{A}{ }_{B} e_{\beta}^{B} .
$$

Реперный формализм нужно обязательно применять, описывая спиноры в римановом пространстве-времени, так как спинорные представления группы Лоренца не могут быть расширены до представлений полной линейной группы. Поэтому спиноры нельзя отнести к координатному локальному базису, а лишь к псевдоортогональному реперному базису. Но ниже реперный формализм применяется для 
другой цели. Считаем, как и ранее, что гравитационное поле не взаимодействует с другими полями.

Чтобы полностью устранить калибровочный произвол, нужно наложить четыре координатных и шесть реперных дополнительных условий. Воспользуемся этой возможностью и устраним лишь часть реперного произвола с помощью трех условий

$$
e_{(0)}^{\mu}(x)=n^{\mu}
$$

где $n^{\mu}(x)$ - нормированная нормаль к поверхности $x^{0}=$ const, проходящей через точку $x$ (см. равенства $(9))$. Здесь и далее реперные индексы берем в скобки, если они записываются в виде чисел, а координатные индексы в этом случае пишем без скобок. Равенство (84) содержит только три условия, так как векторы $n^{\mu}$ и $e_{(0)}^{\mu}$ нормированы. После введения условий (84) теория остается инвариантной относительно полупрямого произведения группы общих преобразований координат на группу трехмерных ортогональных преобразований (т.е. преобразований Лоренца, не изменяющих вектор $\left.e_{(0)}^{\mu}(x)=n^{\mu}(x)\right)$.

На каждой поверхности $x^{0}=$ const вводим переменные АДМ $\beta_{i k} \equiv g_{i k}, N$ и $N^{i}$. Далее, $i, k, \ldots$ - трехмерные координатные индексы $(i, k, \ldots=1,2,3), a, b, \ldots$ трехмерные реперные индексы $(a, b, \ldots=1,2,3)$. В силу условия (84) реперные параметры $e_{A}^{\mu}$ и $e_{\mu}^{A}$ выражаются через $e_{a}^{i}, e_{i}^{a}, N$ и $N^{i}$ следующим образом:

$$
e_{(0)}^{0}=\frac{1}{N}, \quad e_{a}^{0}=0, \quad e_{(0)}^{i}=-\frac{N^{i}}{N}, \quad e_{0}^{(0)}=N, \quad e_{i}^{(0)}=0, \quad e_{0}^{a}=e_{i}^{a} N^{i}
$$

При этом не только

$$
e_{\mu}^{A} e_{A}^{\nu}=\delta_{\mu}^{\nu}
$$

но и

$$
e_{i}^{a} e_{a}^{k}=\delta_{k}^{i}, \quad g_{i k}=\beta_{i k}=e_{i}^{a} e_{k}^{a}, \quad \beta^{i k}=e_{a}^{i} e_{a}^{k}
$$

Полагаем

$$
e \equiv \operatorname{det} e_{i}^{a}
$$

причем

$$
\beta=e^{2}
$$

Имея в виду возможное применение к петлевой теории гравитации, выберем в качестве основных переменных функции

$$
Q_{a}^{i} \equiv e e_{a}^{i}=\sqrt{\beta} e_{a}^{i}, \quad N, \quad N_{i} .
$$

Положим

$$
Q \equiv \operatorname{det} Q_{a}^{i}
$$

причем

$$
Q=\beta,
$$


и определим величины $Q_{i}^{a}$ равенствами

$$
Q_{i}^{a} Q_{a}^{k}=\delta_{i}^{k}
$$

Векторы $Q_{a}^{i}$ (равно как и $e_{a}^{i}$ ) касаются поверхности $x^{0}=$ const. Из соотношений (87) видно, что индексы $a, b, \ldots$ можно поднимать и опускать с помощью символов $\delta^{a b}$ и $\delta_{a b}$. Поэтому нет различия между верхними и нижними индексами $a, b$, и их можно писать, где удобно.

Разовьем канонический формализм на гиперповерхности $x^{0}=$ const в терминах трехмерных переменных $Q_{a}^{i}, N, N^{i}$, обозначая эту гиперповерхность по-прежнему через $\Sigma$. Проще всего можно прийти к цели, начав с формализма ФП (см. раздел 3 ). Согласно формулам (62), (87) и (90)

$$
q^{i k}=\beta \beta^{i k}=\beta e_{a}^{i} e_{a}^{k}=Q_{a}^{i} Q_{a}^{k}
$$

Подставим это выражение в лагранжиан $Ф$ П (67) и будем сначала считать $\pi_{i k}$ и $Q_{a}^{i}$ независимыми. Найдем, что

$$
\pi_{i k} \partial_{0} q^{i k}=2 \pi_{i k} Q_{a}^{k} \partial_{0} Q_{a}^{i}
$$

поэтому переменным $Q_{a}^{i}$ отвечают сопряженные импульсы

$$
\mathcal{P}_{i}^{a}=2 \pi_{i k} Q_{a}^{k}
$$

откуда

$$
\pi_{i k}=\frac{1}{2} Q_{k}^{a} \mathcal{P}_{i}^{a}
$$

Но $\pi_{i k}=\pi_{k i}$, так что возникают новые связи

$$
Q_{k}^{a} \mathcal{P}_{i}^{a}-Q_{i}^{a} \mathcal{P}_{k}^{a}=0
$$

В силу равенств (93) это эквивалентно трем связям

$$
\Phi^{a} \equiv \varepsilon^{a b c} Q^{i b} \mathcal{P}_{i}^{c}=0,
$$

где $\varepsilon^{a b c}$ вполне антисимметрично, $\varepsilon^{123}=1$.

Действие реперного формализма можно записать теперь в следующей канонической форме:

$$
\begin{gathered}
S_{(1)}^{(\text {реп) }}=\int d^{4} x \mathcal{L}_{(1)}^{(\text {реп) }}, \\
\mathcal{L}_{(1)}^{(\text {реп })}=\mathcal{P}_{i}^{a} \partial_{0} Q_{a}^{i}-N \mathcal{H}_{0}-N^{i} \mathcal{H}_{i}-\lambda^{a} \Phi^{a},
\end{gathered}
$$

где мы учли новые связи с помощью множителей Лагранжа $\lambda^{a}$. Предполагается, что в формулах $Ф П(68),(69)$ для $\mathcal{H}_{0}$ и $\mathcal{H}_{i}$ величины $q^{i k}, \pi_{i k}$ выражены через $\mathcal{P}_{i}^{a}, Q_{a}^{i}$ 
согласно $(94),(97)$. Здесь и далее мы для простоты не пишем в $\mathcal{L}_{(1)}^{(\text {реп) }}$ дивергенции, считая, что Вселенная замкнута. При этом

$$
\begin{gathered}
\mathcal{H}_{0}=\frac{1}{4}\left(\frac{2 \varkappa}{\sqrt{Q}}\right)\left(Q_{b}^{k} Q_{b}^{l} \mathcal{P}_{k}^{c} \mathcal{P}_{l}^{c}-\left(Q_{b}^{k} \mathcal{P}_{k}^{b}\right)^{2}\right)-\left(\frac{\sqrt{Q}}{2 \varkappa}\right)(\stackrel{(3)}{R}-2 \Lambda), \\
\mathcal{H}_{i}=Q_{a}^{k}\left(\stackrel{(3)}{\nabla_{k}} \mathcal{P}_{i}^{a}-\stackrel{(3)}{\nabla}_{i} \mathcal{P}_{k}^{a}\right),
\end{gathered}
$$

где, как и ранее, $\stackrel{(3)}{\nabla} k$ - ковариантная производная на гиперповерхности $x^{0}=$ const, содержащая коэффициенты связности $\stackrel{(3)}{\Gamma} \underset{k l}{i}$ и $\stackrel{(3)}{A_{i}{ }^{a} b}$, выраженные через $Q_{a}^{k}$ и $Q_{k}^{a}$.

(3)

Коэффициенты $A_{i}{ }^{a}{ }_{b}$ определяются по аналогии с формулой (80) равенствами

$$
\stackrel{(3)}{A_{k}{ }^{a} b}=e_{i}^{a} \stackrel{(3)}{\Gamma}{ }_{k l}^{i} e_{b}^{l}-e_{i}^{a} \partial_{k} e_{b}^{i} .
$$

Можно проверить, что в силу условия (84)

$$
\stackrel{(3)}{A_{k}{ }^{a} b}=A_{k}{ }^{a},
$$

где $A_{k}{ }^{a}{ }_{b}$ - трехмерная часть четырехмерной связности $A_{\mu}{ }^{A}$. Величины $A_{k}{ }^{a}{ }_{b}$ образуют $S O(3)$-связность. Поэтому

$$
A_{k}{ }^{a}{ }_{b}=A_{k}{ }^{a b}=-A_{k}^{b a}=\varepsilon^{a b c} A_{k}^{c},
$$

где

$$
A_{k}^{c}=\frac{1}{2} \varepsilon^{c a b} A_{k}^{a b}
$$

Из формул (90), (92) следует, что

$$
e_{a}^{i}=Q^{-1 / 2} Q_{a}^{i}, \quad e_{i}^{a}=Q^{1 / 2} Q_{i}^{a} .
$$

Подставляя это в равенства (104) и учитывая выражения (105), (107), находим, что

$$
\begin{aligned}
A_{i}^{c} & =\frac{1}{2} \varepsilon^{c a b}\left(\left(\partial_{k} Q_{i}^{a}-\partial_{i} Q_{k}^{a}\right) Q_{b}^{k}-Q_{a}^{l}\left(\partial_{l} Q_{m}^{d}\right) Q_{b}^{m} Q_{i}^{d}+Q_{i}^{b} Q_{a}^{k} Q_{d}^{l} \partial_{k} Q_{l}^{d}\right)= \\
& =\frac{1}{2} \varepsilon^{c a b}\left(Q_{k}^{a} \partial_{i} Q^{k b}+Q_{i}^{d} Q^{k a}\left(Q_{l}^{b} \partial_{k} Q^{l d}+Q_{l}^{d} \partial_{k} Q^{l b}\right)+Q_{i}^{a} Q^{k b} Q_{l}^{d} \partial_{k} Q^{l d}\right) .
\end{aligned}
$$

Обозначая через $A_{k}$ матрицу $A_{k}^{a b}$, можно определить трехмерную напряженность поля

$$
\stackrel{(3)}{F}_{i k}=\partial_{i} A_{k}-\partial_{k} A_{i}+A_{i} A_{k}-A_{k} A_{i},
$$

причем будут справедливы равенства

$$
\stackrel{(3)}{F}_{i k}^{a b}=\varepsilon^{a b c} \stackrel{(3)}{F}_{i k}^{c}, \quad \stackrel{(3)}{R}_{k, l m}^{i}=e_{a}^{i} \stackrel{(3)}{F}_{l m}^{a b} e_{k}^{b} .
$$


Канонические переменные подчиняются теперь соотношениям

$$
\left\{Q_{a}^{i}, \underset{\sim}{\mathcal{P}_{k}^{b}}\right\}=\delta_{k}^{i} \delta_{b}^{a} \delta^{3}(x-\tilde{x}), \quad\left\{Q_{a}^{i}, \underset{\sim}{Q_{b}^{k}}\right\}=0, \quad\left\{\mathcal{P}_{i}^{b},{\underset{\sim}{\mathcal{P}}}_{k}^{a}\right\}=0 .
$$

Условие симметрии $\pi_{i k}=\pi_{k i}$ в рамках формализма, рассмотренного в данном разделе, имеет место в силу связей (99), в то время как в разделе 3 оно выполнялось по определению. Поэтому скобки Пуассона между $\pi_{i k}$ и $\pi_{l m}$ отличаются от приведенных в разделе 3 . Теперь

$$
\left\{q^{i k}, \underset{\sim}{q^{l m}}\right\}=0, \quad\left\{q^{i k}, \underset{\sim}{\pi_{l m}}\right\}=\delta_{l m}^{i k} \delta^{3}(x-\tilde{x}),
$$

но

$$
\left\{\pi_{i k}, \underset{\sim}{\pi_{l m}}\right\}=\frac{1}{4} Q_{k}^{b} Q_{m}^{b} Q_{i}^{d} Q_{l}^{c} \varepsilon^{c d a} \Phi^{a} \delta^{3}(x-\tilde{x}) .
$$

Поэтому в соотношениях со скобками Пуассона (50)-(52) справа появляются новые члены, но все они пропорциональны связям $\Phi^{a}$. Кроме того,

$$
\left\{\Phi^{a}, \underset{\sim}{\Phi^{b}}\right\}=\varepsilon^{a b c} \Phi^{c} \delta^{3}(x-\tilde{x}), \quad\left\{\Phi^{a}, \underset{\sim}{\mathcal{H}_{0}}\right\}=0, \quad\left\{\Phi^{a}, \underset{\sim}{\mathcal{H}_{i}}\right\}=0 .
$$

Таким образом, классическая алгебра связей замыкается, и все связи $\mathcal{H}_{0}, \mathcal{H}_{i}$ и $\Phi^{a}$ первого рода.

Вместо связей $\mathcal{H}_{i}$ удобно ввести связи

$$
\mathcal{H}_{i}^{\prime} \equiv \mathcal{H}_{i}+A_{i}{ }^{c} \Phi^{c} \equiv Q^{b k}\left(\partial_{k} \mathcal{P}_{i}^{b}-\partial_{i} \mathcal{P}_{k}^{b}\right)+\left(\partial_{k} Q^{k b}\right) \mathcal{P}_{i}^{b}=0
$$

Можно проверить, что величины $\mathcal{H}_{i}^{\prime}$ генерируют преобразования трехмерных координат без изменения реперов как геометрических объектов, а $\Phi^{a}$ - повороты реперов без изменения координат.

Алгебра связей в терминах величин $\mathcal{H}_{0}, \mathcal{H}_{i}^{\prime}, \Phi^{a}$ имеет вид

$$
\begin{aligned}
& \left\{\mathcal{H}_{i}^{\prime}, \mathcal{H}_{\sim}^{\prime}\right\}=\mathcal{H}_{k}^{\prime} \partial_{i} \delta^{3}(x-\tilde{x})-\underset{\sim}{\mathcal{H}_{i}^{\prime}} \partial_{\sim} \delta^{3}(x-\tilde{x}), \\
& \left\{\mathcal{H}_{i}^{\prime}, \mathcal{H}_{\sim}\right\}=\mathcal{H}_{0} \partial_{i} \delta^{3}(x-\tilde{x}),
\end{aligned}
$$

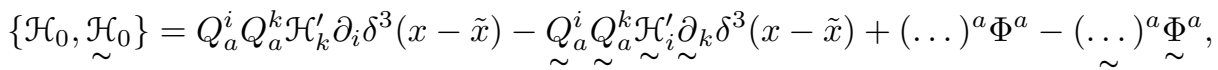

$$
\begin{aligned}
& \left\{\Phi^{a}, \Phi_{\sim}^{b}\right\}=\varepsilon^{a b c} \Phi^{c} \delta^{3}(x-\tilde{x}), \\
& \left\{\Phi^{b}, \mathcal{H}_{\sim}^{\prime}\right\}=-\Phi^{b} \partial_{i} \delta^{3}(x-\tilde{x}), \\
& \left\{\Phi^{a}, \underset{\sim}{\mathcal{H}_{0}}\right\}=0
\end{aligned}
$$

где $(\ldots)^{a}$ - некоторые выражения, составленные из $Q_{a}^{i}$ и $\mathcal{P}_{i}^{a}$.

\section{5. ФОРМАЛИЗМ, ПРИМЕНЯЕМЫЙ В ПЕТЛЕВОЙ ТЕОРИИ ГРАВИТАЦИИ}

Перейдем теперь к каноническому формализму, который лежит в основе петлевой теории гравитации. Можно непосредственно проверить, что трехмерная реперная 
связность (109) допускает представление

$$
A_{i}^{a}=\frac{\delta F}{\delta Q_{a}^{i}(x)},
$$

где

$$
F=\frac{1}{2} \int_{x^{0}=\mathrm{const}} d^{3} x \varepsilon^{a b c} Q_{a}^{l} Q_{k}^{b} \partial_{l} Q^{k c} .
$$

Здесь $x \equiv\left(x^{1}, x^{2}, x^{3}\right)$, а $\delta / \delta Q_{a}^{i}$ - трехмерная вариационная производная. Поскольку

$$
\left\{\mathcal{P}_{i}^{a}(x), f\left[Q_{b}^{k}\right]\right\}=-\frac{\delta f\left[Q_{b}^{k}\right]}{\delta Q_{a}^{i}(x)},
$$

где $f\left[Q_{b}^{k}\right]$ - любой функционал от функций $Q_{a}^{k}(x)$, то

$$
\left\{\mathcal{P}_{m}^{f}, \underset{\sim}{A_{i}^{c}}\right\}=\left\{\underset{\sim}{\mathcal{P}_{i}^{c}}, A_{m}^{f}\right\}
$$

Это позволяет совершить каноническое преобразование

$$
Q_{a}^{i} \longrightarrow Q_{a}^{i}, \quad \mathcal{P}_{i}^{a} \longrightarrow \mathcal{P}_{i}^{\prime a}=\mathcal{P}_{i}^{a}+b A_{i}^{a},
$$

где $b$ - число, именуемое параметром Барберо-Иммирзи. Этому параметру можно придать любое значение. В силу (121) будет выполняться условие

$$
\left\{\mathcal{P}_{i}^{\prime a}, \mathcal{P}_{k}^{\prime b}\right\}=0
$$

Так как $A_{i}^{a}$ зависит лишь от $Q_{i}^{a}$, то будет верным также равенство

$$
\left\{Q_{a}^{i}, \mathcal{P}_{k}^{\prime b}\right\}=\delta_{k}^{i} \delta_{a}^{b} \delta^{3}(x-\tilde{x})
$$

Вслед за (122) можно выполнить еще одну каноническую замену переменных:

$$
\begin{aligned}
Q_{a}^{i} \longrightarrow B_{i}^{a} & =\frac{1}{b} \mathcal{P}_{i}^{\prime a}=A_{i}^{a}+\frac{1}{b} \mathcal{P}_{i}^{a}, \\
\mathcal{P}_{i}^{\prime a} \longrightarrow \Pi_{a}^{i} & =-b Q_{a}^{i} .
\end{aligned}
$$

Величина $B_{i}^{a}$ преобразуется при замене трехмерных координат на гиперповерхности $x^{0}=$ const и реперов, подчиненных все время условию (84), как реперная связность $A_{i}^{a}$. Поэтому контурный интеграл

$$
\operatorname{tr} W(C)=\operatorname{tr} P \exp \left(-\oint_{C} d x^{i} B_{i}\right),
$$

где $B_{i}$ - матрица с элементами $B_{i}^{a b}=\varepsilon^{a b c} B_{i}^{c}$, а интегрирование ведется по замкнутому контуру, лежащему на гиперповерхности $x^{0}=$ const, инвариантен относительно реперных $S O(3)$-преобразований, генерируемых связями $\Phi^{a}$. Это обстоятельство лежит в основе петлевой теории гравитации, в которой в качестве канонических переменных используются величины $B_{i}^{a}$ и $\Pi_{a}^{i}$. 
Из формул (125) следует, что

$$
Q_{a}^{i}=-\frac{1}{b} \Pi_{a}^{i}, \quad \mathcal{P}_{i}^{a}=b\left(B_{i}^{a}-A_{i}^{a}\right),
$$

где

$$
\left.A_{i}^{a} \equiv A_{i}^{a}\right|_{Q_{a}^{i}=-b^{-1} \Pi_{a}^{i}}
$$

Подставив это в действие (101) и учитывая (102) и (103), можно записать результат в двух формах:

$$
\begin{gathered}
S_{1}=\int d^{4} x \mathcal{L}_{1}, \\
\mathcal{L}_{1}=\Pi_{a}^{i} \partial_{0} B_{i}^{a}-N^{\prime} \mathcal{H}_{0}^{\prime}-N^{\prime i} \mathcal{H}_{i}^{\prime}-\lambda^{\prime a} \Phi^{a}= \\
=\Pi_{a}^{i} \partial_{0} B_{i}^{a}-N^{\prime \prime} \mathcal{H}_{0}^{\prime \prime}-N^{\prime \prime} \mathcal{H}_{i}^{\prime \prime}-\lambda^{\prime \prime a} \Phi^{a},
\end{gathered}
$$

где

$$
\begin{aligned}
\Phi^{a} & =-\left(\partial_{i} \Pi_{a}^{i}+\varepsilon^{a b c} B_{i}^{c} \Pi_{b}^{i}\right)=-\sqrt{\Pi} \stackrel{(3 B)}{\nabla}\left(\frac{\Pi_{a}^{i}}{\sqrt{\Pi}}\right) \equiv-D_{i} \Pi_{a}^{i}, \\
\mathcal{H}_{k}^{\prime} & =\mathcal{H}_{k}+A_{k}^{a} \Phi^{a}=-\Pi_{c}^{i} \stackrel{(3)}{F}_{i k}(B)+B_{k}^{b} \Phi^{b}, \\
\mathcal{H}_{0}^{\prime} & =\Pi_{a}^{i} \Pi_{b}^{k} \varepsilon^{a b c} \stackrel{(3)}{F}_{i k}^{c}(B)+4 b^{-3} \frac{1}{(2 \varkappa)^{2}} \Pi\left(\left(1+\varkappa^{2} b^{2}\right) \stackrel{(3)}{R}-2 \Lambda\right),
\end{aligned}
$$

$\mathcal{H}_{k}^{\prime \prime}=\Pi_{c}^{i} \stackrel{(3)}{F}_{i k}^{c}(B)$,

$$
\begin{aligned}
\mathcal{H}_{0}^{\prime \prime}= & \Pi_{a}^{i} \Pi_{b}^{k} \varepsilon^{a b c} \stackrel{(3)}{F}_{i k}^{c}(B)+ \\
& +\left(1+\varkappa^{2} b^{2}\right)\left(\Pi_{b}^{k} \Pi_{b}^{l}\left(B_{k}^{c}-A_{k}^{c}\right)\left(B_{l}^{c}-A_{l}^{c}\right)-\left(\Pi_{b}^{k}\left(B_{k}^{b}-A_{k}^{b}\right)\right)^{2}\right)+2 b^{-1} \Lambda \Pi .
\end{aligned}
$$

Здесь

$$
\begin{aligned}
& N^{\prime}=\frac{N}{4}\left(\frac{2 \varkappa}{\sqrt{Q}}\right), \quad N^{\prime k}=N^{k}, \\
& \lambda^{\prime a}=\lambda^{a}-\frac{N}{4}\left(\frac{2 \varkappa}{\sqrt{Q}}\right) Q^{k d} \mathcal{P}_{k}^{b} \varepsilon^{d b a}-N^{k} A_{k}^{b}+b \varkappa e_{a}^{i} \partial_{i} N, \\
& N^{\prime \prime}=-\frac{N}{(2 \varkappa) b^{2} \sqrt{Q}}, \quad N_{k}^{\prime \prime}=-N_{k}, \\
& \lambda^{\prime \prime a}=\lambda^{a}+\frac{1}{2} N^{k} \mathcal{P}_{k}^{a}-N\left((2 \varkappa) b^{2} \sqrt{Q}\right)^{-1} Q_{d}^{k} \mathcal{P}_{k}^{f} \varepsilon^{d f a}+2((2 \varkappa) b)^{-1} e_{a}^{i} \partial_{i} N \\
& \Pi=\operatorname{det} \Pi_{a}^{i}, \quad A_{k}^{a}=\left.A_{k}^{a}\left(Q_{a}^{i}\right)\right|_{Q_{a}^{i}=-b^{-1} \Pi_{a}^{i}}, \quad \stackrel{(3)}{R}=\left.\stackrel{(3)}{R}\left(Q_{b}^{i}\right)\right|_{Q_{b}^{i}=-b^{-1} \Pi_{b}^{i}}, \\
& F_{i k}^{(3)}(B)=\partial_{i} B_{k}-\partial_{k} B_{i}+B_{i} B_{k}-B_{k} B_{i},
\end{aligned}
$$

(3)

$B_{i}, \stackrel{F}{F}_{i k}-$ матрицы с элементами

$$
B_{i}^{a b}=\varepsilon^{a b c} B_{i}^{c}, \quad \stackrel{(3)}{F}_{i k}^{a b}=\varepsilon^{a b c} \stackrel{(3)}{F}_{i k}^{c},
$$


a $\stackrel{(3 B)}{\nabla}{ }_{i}-$ трехмерная ковариантная производная со связностью $B_{i}^{a b}$.

Видно, что выражения для $\mathcal{H}_{0}^{\prime}$ и $\mathcal{H}_{0}^{\prime \prime}$ резко упрощаются и становятся равными, если продолжить теорию в комплексную область и положить

$$
b=\mp i \varkappa .
$$

Тогда согласно (125)

$$
B_{i}^{c}=A_{i}^{c} \pm i \varkappa \mathcal{P}_{i}^{c}
$$

Но в реперном базисе, в котором $e_{(0)}^{\mu}=n^{\mu}$, справедливы равенства

$$
A_{i}^{(0) c}=N \Gamma_{i k}^{0} e_{c}^{k}=-K_{i k} e_{c}^{k}
$$

причем

$$
\pi_{i k}=\frac{1}{(2 \varkappa) \sqrt{\beta}} K_{i k}, \quad \mathcal{P}_{i}^{a}=2 \pi_{i k} Q_{a}^{k}=\frac{1}{\varkappa} K_{i k} e_{a}^{k}=-\frac{1}{\varkappa} A_{i}^{(0) c},
$$

вследствие чего равенство (137) принимает вид

$$
B_{i}^{c}=A_{i}^{c} \mp i A_{i}^{(0) c},
$$

T.e.

$$
B_{i}^{a b}=A_{i}^{a b} \mp i \varepsilon^{a b c} A_{i}^{(0) c}
$$

Можно ввести поля

$$
A_{\mu}^{A B( \pm)}=A_{\mu}^{A B} \mp \frac{i}{2} \eta^{A D} \eta^{B E} \varepsilon_{D E F H} A_{\mu}^{F H},
$$

откуда

$$
A_{i}^{a b( \pm)}=A_{i}^{a b} \mp i \varepsilon^{a b c} A_{i}^{(0) c} .
$$

Поля $A_{\mu}^{A B( \pm)}$ удовлетворяют условиям самодуальности (антисамодуальности)

$$
A_{\mu}^{A B( \pm)}=\mp \frac{i}{2} \eta^{A D} \eta^{B E} \varepsilon_{D E F H} A_{\mu}^{F H( \pm)},
$$

причем

$$
A_{\mu}^{A B}=A_{\mu}^{A B(+)}+A_{\mu}^{A B(-)} .
$$

Таким образом, выражения для $\mathcal{H}_{0}^{\prime}, \mathcal{H}_{0}^{\prime \prime}$ упрощаются, если

$$
B_{\mu}^{a b}=A_{\mu}^{a b(+)}
$$

или

$$
B_{\mu}^{a b}=A_{\mu}^{a b(-)} .
$$

В этом случае все связи полиномиально зависят от переменных $B_{i}^{a}, \Pi_{a}^{i}$. Но для возвращения в вещественную область нужно удовлетворить сложному условию

$$
B_{i}^{a}+B_{i}^{a *}=\left.2 A(Q)\right|_{Q_{a}^{i}=-b^{-1} \Pi_{a}^{i}}
$$


где * обозначает комплексное сопряжение в классическом случае и эрмитово сопряжение после квантования. Из-за сложности условия (148) в настоящее время предпочитают строить петлевую теорию гравитации при вещественном значении параметра $b$, когда связь $\mathcal{H}_{0}^{\prime}$ (или $\left.\mathcal{H}_{0}^{\prime \prime}\right)$ весьма сложна.

Изложенные выше классические канонические формулировки теории гравитации нашли и продолжают находить применение при работе над проблемой квантования этой теории.

Благодарности. Автор выражает благодарность региональному бюро ЮНЕСКО по научному сотрудничеству в Европе за поддержку Международной школы физики им. В. А. Фока.

\section{Список литературы}

[1] A. Ashtekar, J. Lewandowski, Class. Q. Grav., 21 (2004), R53; gr-qc/0404018.

[2] R. Arnowitt, S. Deser, C. W. Misner, "The Dynamics of General Relativity", Gravitation: an introduction to current research, ed. L. Witten, Wiley, N.Y., 1962, Ch. 7, p. 227; gr-qc/0405109.

[3] В. Н. Попов, Л. Д. Фаддеев, УФН, 111 (1973), 427.

[4] Н. П. Коноплева, В. Н. Попов, Калибровочные поля, УРСС, М., 2000.

[5] М. Грин, Дж. Шварц, Э. Виттен, Теория суперструн, т. 1, 2, Мир, М., 1990. 